\title{
Efforts to Reach More Children with Effective Vaccines Through Routine Immunization in The WHO African Region: 2013-2015
}

\author{
Blanche Anya*, Joseph Okeibunor, Richard Mihigo, Alain Poy, Felicitas Zawaira
}

WHO Regional Office for Africa, Brazzaville, Congo

Article Info

\section{Article Notes}

Published: August 02, 2018

\section{${ }^{*}$ Correspondence:}

Dr. Blanche Anya, WHO Regional Office for Africa, Brazzaville, Congo; Telephone no: +242 06887 1950; Email: anyab@who.int

(c) 2018 Blanche A. This article is distributed under the terms of the Creative Commons Attribution 4.0 International License.

\section{Keywords:}

Routine Immunization

Vaccines

African Region

\section{ABSTRACT}

Background: Some progress has been made in expanding immunization in the African Region over the last four decades. However, an estimated $22 \%$ of the eligible children in the African Region, located in four countries of the African Region (Democratic Republic of the Congo, Ethiopia, Nigeria and South Africa), continue to miss vaccination services for various reasons. This paper documents the status of routine immunization in the African Region.

Methods: Programme records, reports and statistics were reviewed for this paper.

Results: Challenges remain in reaching an estimated $20-30 \%$ of children across the Region. In addition to the traditional vaccines (DTP, measles, polio and tuberculosis) newer ones, such as for Pneumococcal conjugate vaccine (PCV) and rotavirus, are being rolled out in the Region but uptake and coverage are slow and patchy both within and between countries.

Conclusion: The new regional strategic plan for immunization 2014-2020 is intended to provide policy and programmatic guidance to Member States, in line with the 2011-2020 Global Vaccine Action Plan (GVAP), in order to optimize immunization services and assist countries to further strengthen their immunization programmes.

\section{Introduction}

Vaccination remains one of the greatest public health accomplishments in protecting lives. It has been credited with significant reduction incidence of most vaccine-preventable diseases, therefore contributing to the reduction of morbidity and mortality due to vaccine preventable disease ${ }^{1-6}$. Smallpox, a human and disease and rinderpest, which affect livestock, were eliminated through broad immunization with effective vaccines ${ }^{7}$. In the African Region, immunization programmes have recorded remarkable successes in delivering effective vaccines and ensuring equitable vaccination of the populations irrespective of social location, class, ethnicity or religion. Immunization coverage rates have shown upward trends over the years and access has increased remarkably even in previously hard to reach populations. Following global, regional and national efforts in the promotion of immunization, DTP3 coverage, the acknowledged indicator of the performance of immunization programmes, rose from $5 \%$ in 1980 to $76 \%$ in 2015 in the African Region ${ }^{8-10}$.

There has also been significant progress in the introduction of new and under-utilized vaccines in Africa ${ }^{10}$. Currently, many more children and adolescents receive vaccines to prevent diseases 
associated with childhood and adolescence, such as pneumonia, diarrhoea, yellow fever, measles and human papillomavirus (HPV). By 2012, 81\% (27/33) of the countries at the risk for yellow fever had introduced the vaccination into their Expanded Programme on Immunization (EPI) schedules ${ }^{11}$, compared to $27 \%(9 / 33)$ in $2000^{9}$.

Pneumococcal conjugate vaccine (PCV) and rotavirus vaccines had been introduced in 37 and 29 of the 47 Member States as of the end of 2015, respectively. Meningitis A vaccine has been introduced since 2010 in campaigns in 16 countries of the meningitis belt in the African Region, with nearly 260,000,000 persons vaccinated since its introduction (WHO AFRO database 2015). Surveillance for diseases targeted by these new vaccines has been strengthened in all countries as part of monitoring disease trends and vaccine impact.

The strengthened disease surveillance has shown a decrease in the burden of vaccine-preventable diseases (VPD) as illustrated by the decline in the incidence of meningitis due to Neisseria meningitidis serotype A. As of December 2015 there has been no laboratory confirmed case of meningococcal A meningitis among vaccinated persons. Therefore, the Region is on course towards achieving the goal of eliminating meningococcal meningitis A outbreaks as a major public health problem.

Associated with immunization also is the eradication, elimination and control of VPDs in the Region. A clear case is the successful interruption of wild polioviruses (WPV) transmission in the Region ${ }^{12}$. With the use of effective vaccines, the last reported cases of the WPV types 2 and 3 were made in 1998 and 2012, respectively. Between 2000 and 2014, the number of African countries endemic with the WPV1 decreased from 12 to one. New WPV cases reported in 2012 decreased by $63 \%$, with 128 cases in four countries (Nigeria, Cameroon, Guinea and Ethiopia) compared to 350 cases in 12 countries in $2011^{13}$. By July 2014, Nigeria, the only country endemic with the transmission of WPV in the Region, recorded its last WPV case and was removed from the list of endemic countries in 2015.

Similar successes have been recorded against other VPDs. This is the case for measles and maternal and neonatal tetanus elimination. As of December 2015, a total of $36 / 47$ countries in the region had eliminated maternal and neonatal tetanus.

There has been $78 \%$ decline in measles case reports since 2000, from a reported number of 520,102 in the year 2000 to 113,938 in 2015. (African Regional Measles Elimination Status Report, August 2016, IVD, AFRO). However, there was a sharp increase in reported cases between the years 2010 - 2011, owing to the various outbreaks reported across the Region, especially in southern African countries. These outbreaks were mainly driven by a shift of epidemiological susceptibility to older age groups. The increase in the case reports noted in 2012 and 2013 represents large outbreaks documented in DR Congo and Nigeria, which contributed 88,381 and 52,852 cases respectively in 2013 , amounting to $83 \%$ of the total 171,178 cases reported in that particular year. Between 2000 and 2014, the estimated reduction in measles deaths has been $86 \%$ for the African region, from an estimated 342,000 deaths in 2000 (95\% confidence interval between $225,400-574,200$ ) to an estimated 48,000 deaths in 2014 ( $95 \%$ confidence interval between 41,600 - 165,000).

This paper documents progress made in the delivery of a wide range of vaccines in the Region using strategies enshrined in the Global Vaccine Action Plan (GVAP) as well as the Regional Immunization Strategic Plan (RISP): 20142020 .

\section{Methods}

We conducted detailed review of country WHO and UNICEF estimates of immunization coverage data released in July 2015, as the major data source for this paper. We also reviewed programmes reports and records over the period. The analysis employed mainly descriptive statistics to show trends in coverage with the different vaccine antigens in the Region. Tables and figures were also used to illustrate some of the findings in the review.

\section{Coverage during the period 2013-2015}

The 2015 World Health Organization (WHO) and United Nations Children's Fund (UNICEF) Estimates of National Immunization Coverage (WUENIC) revealed that Regional coverage for DTP3 containing vaccine increased from $76 \%$ to $77 \%$ between 2014 and 2015 . An estimated 24.96 million children were vaccinated with DTP3 in 2015 compared to 23.98 million in 2014. A total of 16 countries reached $\geq 90 \%$ coverage compared to 18 in 2014, and coverage increased in 17 countries including in four of the six priority countries (Chad, Ethiopia, the Democratic Republic of Congo [DRC], and Nigeria). Similarly, two of the countries, Liberia and Sierra Leone, which experienced recent disruption of their public health systems due to Ebola virus outbreak increased their coverage. Other countries are (Benin, Cote d'Ivoire, Eritrea, Ethiopia, Gabon, Gambia, Mozambique, Namibia, Nigeria, Sao Tome, Togo, Tanzania and Zambia (Figure 1 and 2). However, a decrease in the coverage was recorded in 16 countries, including the countries with disruption of their health systems due to persistent internal conflict like South Sudan. Others, such as Madagascar and Kenya, had various challenges including poor implementation of the Reach Every District (RED) approach and effects of recent devolution of governance, respectively. The coverage in Chad, Central Africa Republic 


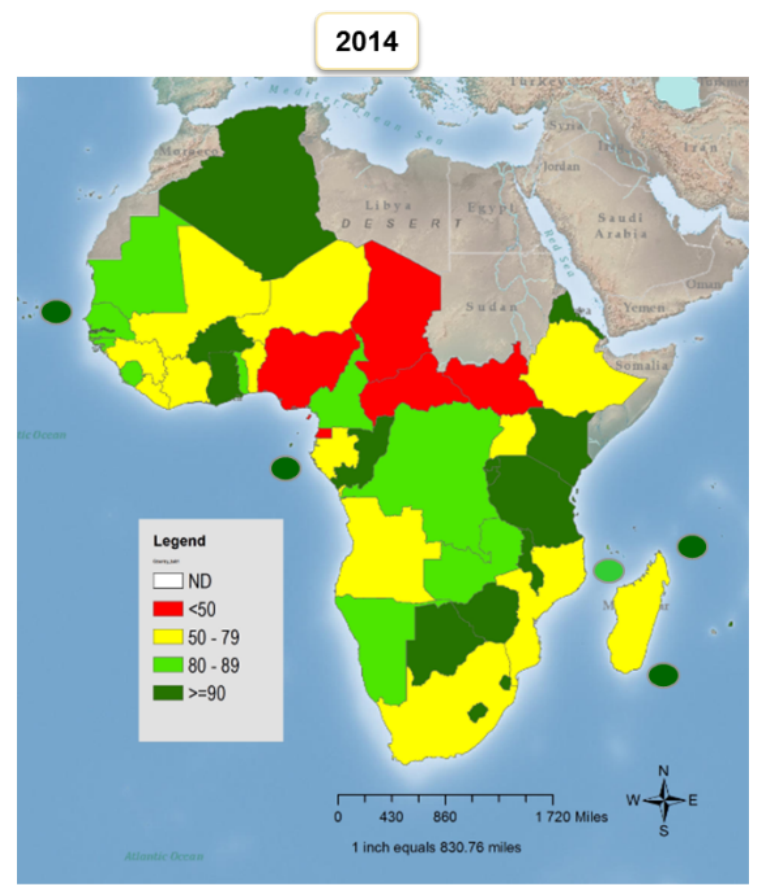

Regional Cov=74\%

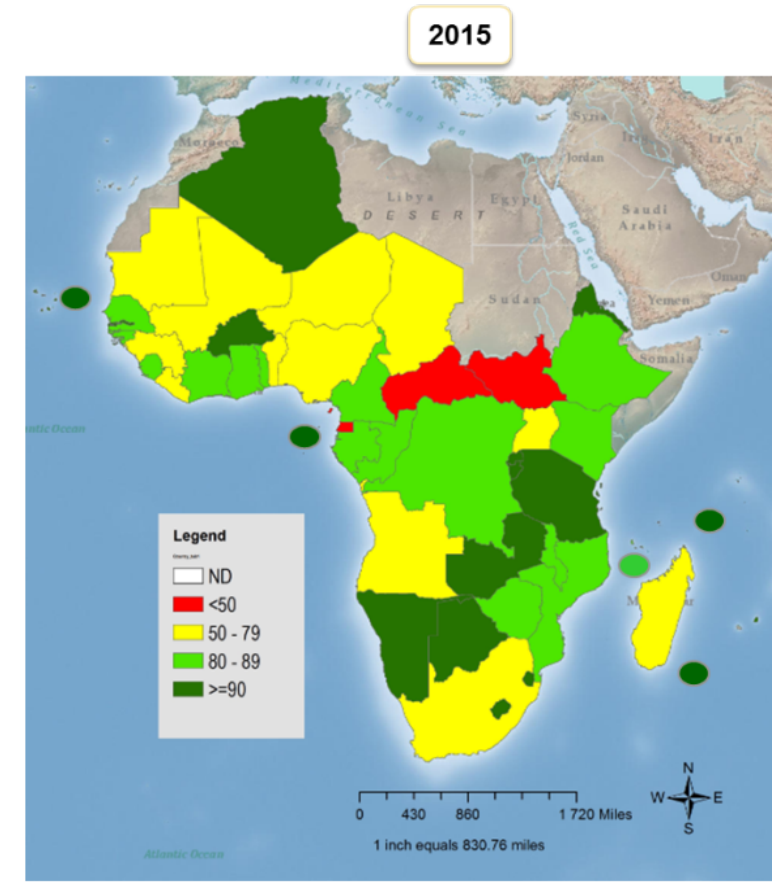

Regional Cov=76\%

Source: WHO/UNICEF estimates for 2015, July 2016 release

http://apps.who.int/immunization_monitoring/globalsummary/timeseries/tswucoveragedtp3.html

Figure 1: Regional coverage of $3^{\text {rd }}$ dose of Diphtheria Tetanus Pertussis Containing vaccine 2014 and 2015

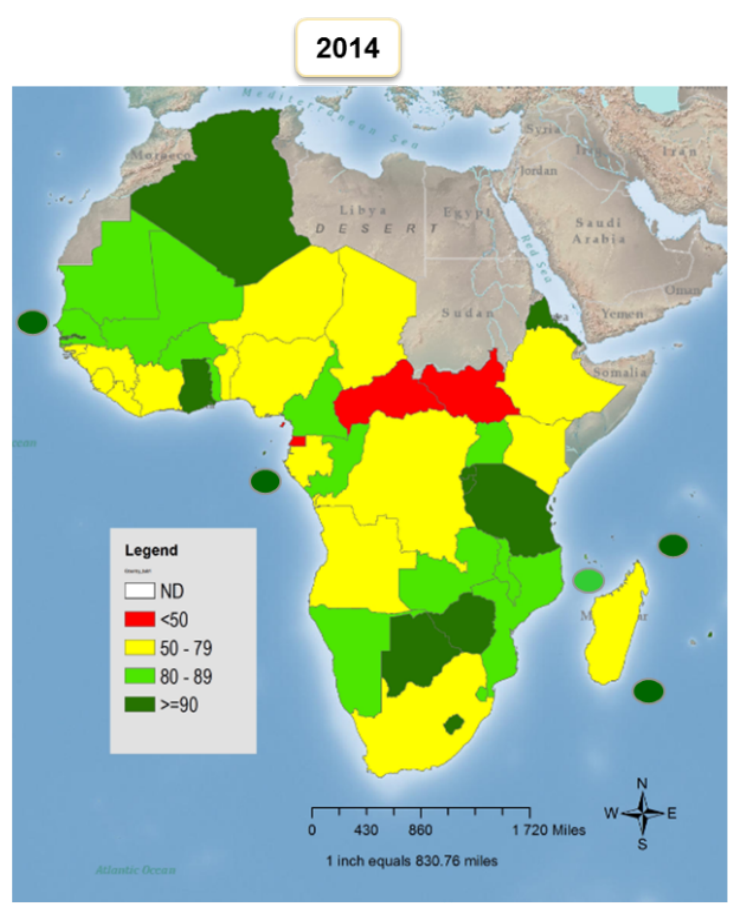

Regional Cov=72\%

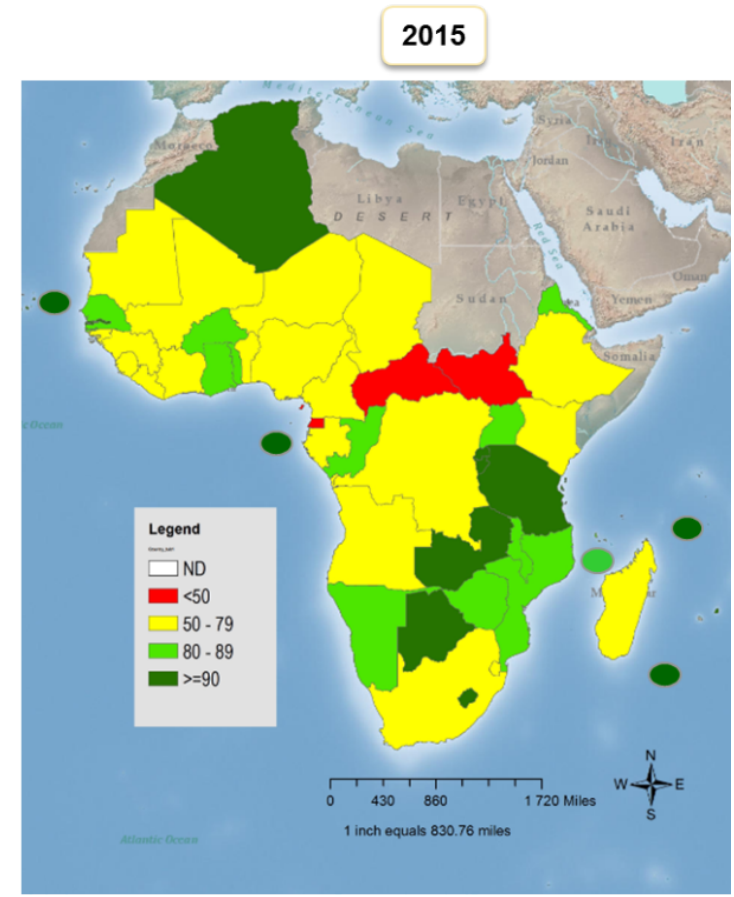

Regional Cov=74\%

Figure 2: Regional coverage of 1st dose Measles Containing Vaccine (MCV1) 2014-2015. 
(CAR), Equatorial Guinea and South Sudan remained below $50 \%$ for both years.

A comparative analysis of the 2015 DTP3 coverage, using administrative data from Countries Joint Reported Form (JRF) and WHO-UNICEF Estimates of National Immunization Coverage (WUENIC), revealed similar coverage in 27 countries. The WUENIC for Botswana, Kenya and Lesotho was higher than the administrative data highlighting possible underreporting from the national administrative system. A comparison of the two datasets in 17 countries revealed gaps between these two sources of data with administrative data reporting higher coverage than what was seen in WUENIC. The highest gap $(\geq 20$ points) was observed in Chad, Niger, South Africa, and Mali. Moderate gap ( $>10$ and $<20$ ) was observed in South Sudan, Nigeria, Madagascar, DRC, Liberia, Ethiopia, Guinea Bissau and Uganda. Minor gap ( $\geq 3$ and $<10$ ) was observed in Guinea, Zimbabwe, Cote d'Ivoire.

Furthermore, the data revealed that an estimated 7.84 million children were not reached with DTP3 in 2015 compared to 8.2 million in 2014, translating into about 4.3 percentage point reduction in the number of unreached children between the two periods. Approximately $75 \%$ of these children are located in 10 countries, with $57 \%$ in 5 countries - Nigeria, DRC, Ethiopia, Angola and Uganda as shown in Figure 3. Five of the ten countries with a high number of unreached children are among the priority countries in the African Region, which are being supported in the development and implementation of national immunization coverage improvement plans with specific strategies to reach the unreached children. These are collaborative efforts with other immunization partners.

Similar to the increase in DTP3 coverage, coverage with $1^{\text {st }}$ dose of Measles-Containing Vaccine (MCV1) and $3^{\text {rd }}$ dose of oral polio (OPV3) vaccines have been on a steady rise since 1980 (Figure 4). On immunization against measles, the Region achieved a significant increase in the first dose measles vaccination (MCV1) coverage between 2001 (54\% MCV1 coverage WUENIC) and 2009 (73\% MCV1 coverage WUENIC). However, Regional MCV1 coverage levels stagnated around $71-74 \%$ between the years 2009- 2015. This trend is also reflected in the number of countries achieving $90 \%$ or more coverage, which increased from only 4 in 2000 to 17 in 2010. In 2015 only 12 countries achieved or maintained MCV1 coverage of $90 \%$ or more. Only 12 countries in the Region reached $90 \%$ coverage for the first dose of MCV1 while 11 countries sustained $90 \%$ coverage during the 2 years. These include Algeria, Botswana, Burundi, Cape Verde, Gambia, Lesotho, Mauritius, Rwanda, Sao Tome et Principe, Seychelles, Tanzania, and Zambia. In 16 countries, MCV1 coverage decreased between 2014 and 2015 with a significant decrease ( $>5$ points) in 7 countries. Three of these countries (CAR, Equatorial Guinea, and South Sudan) reported coverage below $50 \%$ for both years.

Moreover, few of the countries with large populations, as well as the countries with challenging infrastructure/ programme gaps like Ethiopia, DR Congo, Nigeria, Chad, Madagascar, South Africa, have not been able to improve MCV1 coverage levels in the past 5 years substantially. The MCV1 coverage levels for the years 2011 - 2015 (WUENIC)

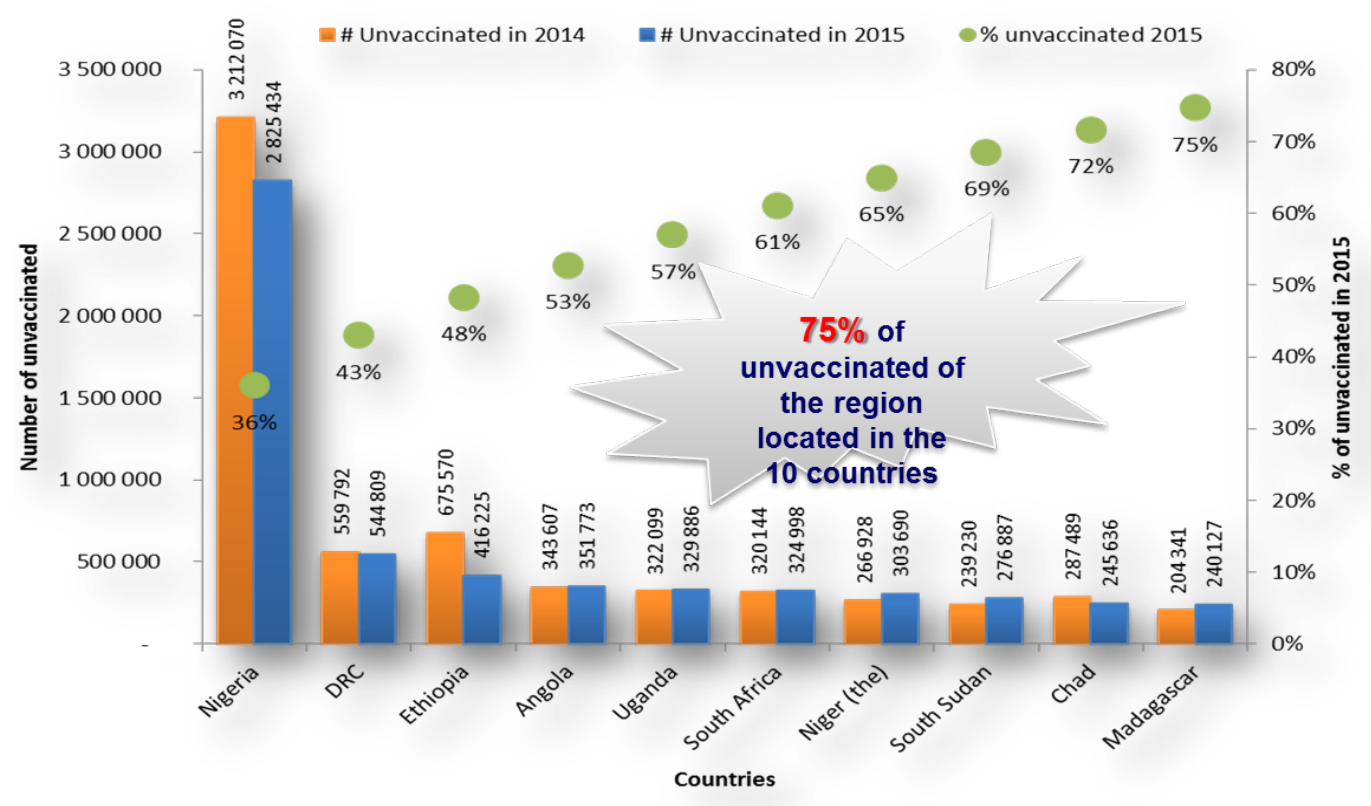

Figure 3: Countries with highest number of DTP3 unimmunized children, 2014-2015 


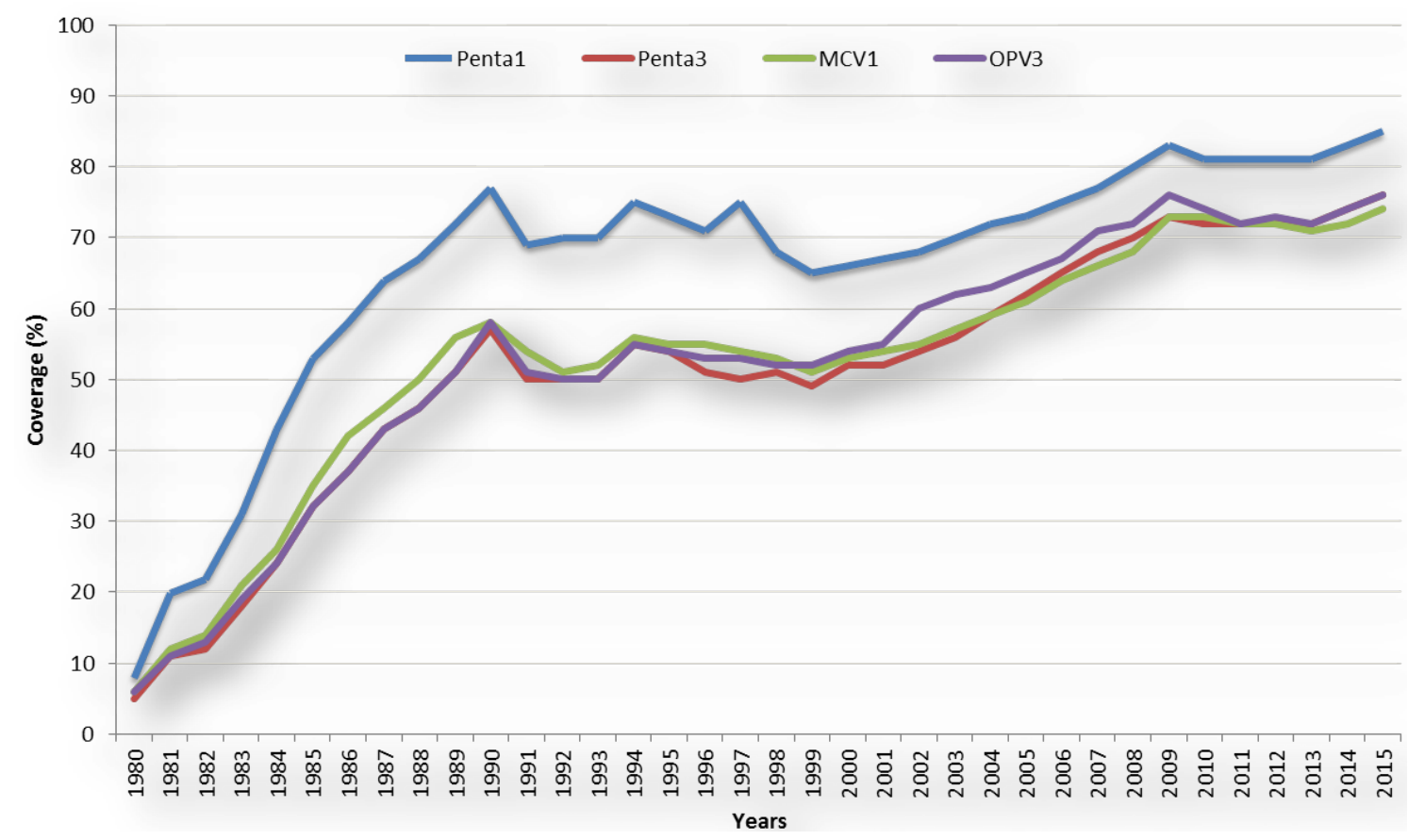

Figure 4: Regional coverage with $1^{\text {st }}$ and $3^{\text {rd }}$ dose of Diphtheria, Tetanus Pertussis containing vaccine (DTP3), $3^{\text {rd }}$ dose of Oral Polio Vaccine ()OPV3, $1^{\text {st }}$ dose of Measles Containing Vaccine, (MCV1) and $1980-2015$

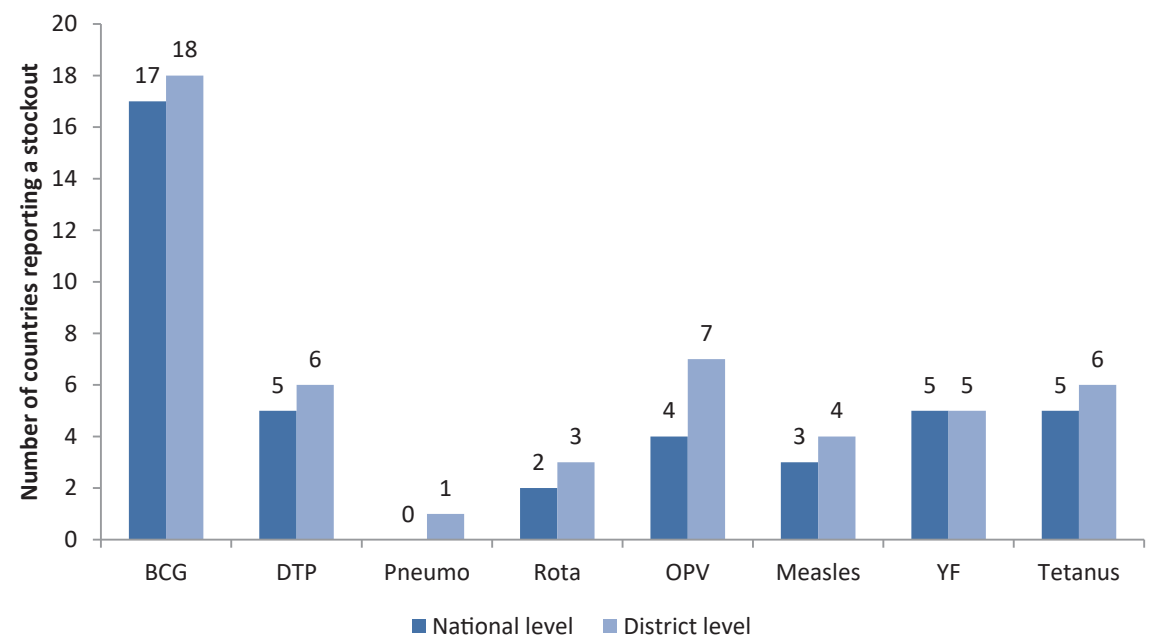

Figure 5: Number of countries that reported vaccine stockouts (in weeks) at the national and district levels in 2015

in the six countries that make up half (53\%) of the Region's population shows that only Tanzania has managed to attain and maintain high coverage.

An analysis of coverage data on all antigens in the Region is shown in Table 1 . The Region is seen to be attaining below $90 \%$ coverage on all nine antigens reported for 2015 . However, unlike in the past, some countries in the Region, such as Algeria and Rwanda have achieved the objective of $\geq 90 \%$ on all antigens delivered in the country. Other countries, such as Burundi, Gambia, Ghana, Mauritius and Sao Tome and Principe, have achieved the objectives of $\geq 90 \%$ national coverage on all but one antigen.
Failure to meet the vaccination objectives is attributed in part to vaccine stock-outs. Figure 5 shows that in the 2015 JRF, two-fifths of the countries (19/47) in the Region reported at least one episode of stock-out for a duration of at least one week. BCG vaccine has been the most affected antigen for stock-out (19 countries), followed by OPV and DTP-containing vaccine and Tetanus toxoid (6 countries). Most of the stockouts at district level were due to unavailability of vaccines at the national level. Stock-outs at the district level have probably contributed to the interruption of vaccination at peripheral levels and consequently to the non-achievements of the set objectives in many countries. 
Table 1: Regional coverage on all antigens by country for 2015

\begin{tabular}{|c|c|c|c|c|c|c|c|c|c|c|c|c|c|c|c|c|c|c|c|c|c|c|}
\hline \multirow{2}{*}{ Country } & \multicolumn{2}{|c|}{ BCG } & \multicolumn{2}{|c|}{ Penta 1} & \multicolumn{2}{|c|}{ Penta 3} & \multicolumn{2}{|c|}{ MCV1 } & \multicolumn{2}{|c|}{ MCV2 } & \multicolumn{2}{|c|}{ PAB } & \multicolumn{2}{|c|}{ PCV3 } & \multicolumn{2}{|c|}{ OPV3 } & \multicolumn{2}{|c|}{ RCV1 } & \multicolumn{2}{|c|}{ Rota Last } & \multicolumn{2}{|c|}{ YFV } \\
\hline & 2015 & 2014 & 2015 & 2014 & 2015 & 2014 & 2015 & 2014 & 2015 & 2014 & 2015 & 2014 & 2015 & 2014 & 2015 & 2014 & 2015 & 2014 & 2015 & 2014 & 2015 & 2014 \\
\hline Algeria & 99 & 99 & 99 & 99 & 95 & 95 & 75 & 68 & 99 & 99 & 92 & 92 & & & 95 & 95 & & & & & & \\
\hline Angola & 79 & 81 & 77 & 81 & 64 & 64 & 97 & 97 & 26 & & 78 & 78 & 58 & 45 & 70 & 68 & & & 49 & 18 & 72 & 77 \\
\hline Benin & 89 & 93 & 90 & 90 & 79 & 75 & 88 & 88 & & & 85 & 93 & 74 & 70 & 79 & 75 & & & & & 71 & 64 \\
\hline Botswana & 98 & 98 & 98 & 98 & 95 & 95 & 93 & 94 & 85 & 85 & 92 & 92 & 81 & 81 & 96 & 96 & & & 82 & 82 & & \\
\hline Burkina Faso & 98 & 98 & 95 & 95 & 91 & 91 & 92 & 93 & 50 & 17 & 92 & 89 & 91 & 91 & 91 & 91 & 68 & & 91 & 91 & 88 & 88 \\
\hline Burundi & 93 & 92 & 97 & 98 & 94 & 95 & 79 & 80 & 65 & 60 & 85 & 85 & 94 & 95 & 94 & 95 & & & 96 & 96 & & \\
\hline Cabo Verde & 94 & 99 & 97 & 99 & 93 & 95 & 49 & 49 & 95 & 79 & 92 & 92 & & & 93 & 95 & 95 & 79 & & & & \\
\hline Cameroon & 74 & 82 & 92 & 93 & 84 & 87 & 62 & 54 & & & 85 & 85 & 85 & 87 & 83 & 86 & & & 73 & 46 & 77 & 80 \\
\hline $\begin{array}{l}\text { Central African } \\
\text { Republic (the) }\end{array}$ & 74 & 74 & 69 & 69 & 47 & 47 & 81 & 80 & & & 60 & 60 & 47 & 47 & 47 & 47 & & & & & 48 & 48 \\
\hline Chad & 70 & 59 & 60 & 60 & 55 & 46 & 80 & 80 & & & 75 & 60 & & & 62 & 54 & & & & & 49 & 40 \\
\hline Comoros (the) & 73 & 76 & 81 & 83 & 80 & 80 & 72 & 62 & & & 85 & 85 & & & 81 & 79 & & & & & & \\
\hline Congo (the) & 85 & 95 & 85 & 95 & 80 & 90 & 79 & 77 & & & 85 & 85 & 80 & 85 & 80 & 90 & & & 80 & 60 & 65 & 65 \\
\hline Côte d'Ivoire & 79 & 84 & 99 & 93 & 83 & 76 & 27 & 44 & & & 85 & 82 & 72 & & 81 & 76 & & & & & 49 & 49 \\
\hline $\begin{array}{l}\text { Democratic } \\
\text { Republic of the } \\
\text { Congo (the) }\end{array}$ & 74 & 78 & 82 & 81 & 81 & 80 & 85 & 90 & & & 82 & 82 & 73 & 61 & 78 & 79 & & & & & 65 & 65 \\
\hline Equatorial Guinea & 48 & 56 & 28 & 59 & 16 & 20 & 78 & 70 & & & 70 & 70 & & & 17 & 24 & & & & & & \\
\hline Eritrea & 97 & 97 & 98 & 97 & 95 & 94 & 68 & 61 & 75 & & 94 & 94 & & & 95 & 94 & & & 96 & 25 & & \\
\hline Ethiopia & 75 & 75 & 94 & 86 & 86 & 77 & 97 & 96 & & & 80 & 80 & 85 & 76 & 85 & 75 & & & 83 & 63 & & \\
\hline Gabon & 98 & 91 & 87 & 77 & 80 & 70 & 89 & 92 & & & 85 & 85 & & & 79 & 68 & & & & & 68 & 60 \\
\hline Gambia (the) & 98 & 96 & 99 & 98 & 97 & 96 & 52 & 52 & 77 & 73 & 92 & 92 & 97 & 96 & 96 & 97 & & & 97 & 92 & 97 & 96 \\
\hline Ghana & 97 & 99 & 97 & 99 & 88 & 98 & 69 & 69 & 63 & 67 & 88 & 88 & 88 & 93 & 88 & 93 & 89 & 92 & 88 & 98 & 88 & 92 \\
\hline Guinea & 72 & 72 & 60 & 60 & 51 & 51 & 75 & 79 & & & 80 & 80 & & & 42 & 42 & & & & & 53 & 53 \\
\hline Guinea-Bi & 94 & 94 & 92 & 92 & 80 & 80 & 90 & 90 & & & 80 & 80 & 10 & & 78 & 78 & & & & & 69 & 53 \\
\hline Kenya & 87 & 94 & 96 & 97 & 89 & 92 & 64 & 58 & 28 & & 80 & 76 & 75 & 81 & 83 & 93 & & & 66 & 19 & 1 & 1 \\
\hline Lesotho & 98 & 98 & 98 & 98 & 93 & 93 & 58 & 64 & 82 & 82 & 83 & 83 & 29 & & 90 & 90 & & & & & & \\
\hline Liberia & 74 & 73 & 77 & 74 & 52 & 50 & 87 & 85 & & & 89 & 89 & 56 & 45 & 52 & 49 & & & & & 56 & 54 \\
\hline Madagascar & 70 & 75 & 79 & 83 & 69 & 73 & 76 & 80 & & & 78 & 78 & 69 & 72 & 71 & 73 & & & 69 & 39 & & \\
\hline Malawi & 90 & 97 & 93 & 97 & 88 & 91 & 95 & 95 & 8 & & 89 & 89 & 88 & 87 & 88 & 87 & & & 84 & 83 & & \\
\hline Mali & 79 & 79 & 80 & 90 & 68 & 77 & 55 & 60 & & & 85 & 85 & 58 & 78 & 76 & 84 & & & 33 & 13 & 64 & 64 \\
\hline Mauritania & 85 & 98 & 87 & 88 & 73 & 84 & 70 & 84 & & & 80 & 80 & 71 & 71 & 67 & 84 & & & 56 & 5 & & \\
\hline Mauritius & 98 & 97 & 98 & 97 & 97 & 97 & 99 & 98 & 85 & 85 & 95 & 95 & & & 98 & 98 & 99 & 98 & 66 & & & \\
\hline Mozambique & 95 & 94 & 90 & 92 & 80 & 79 & 85 & 85 & & & 83 & 83 & 80 & 73 & 80 & 79 & & & 17 & & & \\
\hline Namibia & 94 & 97 & 98 & 92 & 92 & 88 & 85 & 83 & & & 85 & 85 & 81 & & 92 & 88 & & & 87 & & & \\
\hline Niger (the) & 77 & 76 & 85 & 89 & 65 & 68 & 73 & 72 & 16 & 3 & 81 & 81 & 74 & 13 & 65 & 67 & & & 70 & 19 & 72 & 70 \\
\hline Nigeria & 68 & 64 & 70 & 64 & 56 & 49 & 54 & 51 & & & 55 & 55 & 13 & & 55 & 49 & & & & & 54 & 51 \\
\hline Rwanda & 99 & 99 & 99 & 99 & 98 & 98 & 97 & 97 & 87 & & 90 & 90 & 98 & 98 & 98 & 98 & 97 & & 98 & 98 & & \\
\hline $\begin{array}{l}\text { Sao Tome and } \\
\text { Principe }\end{array}$ & 97 & 95 & 98 & 98 & 96 & 95 & 93 & 92 & 76 & 71 & 99 & 99 & 96 & 95 & 96 & 95 & & & & & 93 & 92 \\
\hline Senegal & 95 & 95 & 94 & 94 & 89 & 89 & 80 & 80 & 54 & 13 & 91 & 91 & 89 & 81 & 85 & 85 & 80 & 80 & 83 & & 80 & 80 \\
\hline Seychelles & 99 & 98 & 99 & 99 & 97 & 99 & 98 & 99 & 98 & 98 & 99 & 99 & & & 97 & 99 & 98 & 99 & & & & \\
\hline Sierra Leone & 90 & 90 & 95 & 88 & 86 & 83 & 76 & 78 & 60 & & 85 & 85 & 86 & 83 & 86 & 83 & & & 85 & 53 & 78 & 80 \\
\hline South Africa & 69 & 77 & 72 & 73 & 69 & 70 & 76 & 70 & 63 & 60 & 80 & 80 & 69 & 65 & 70 & 71 & & & 72 & 72 & & \\
\hline South Sudan & 43 & 46 & 49 & 49 & 31 & 39 & 20 & 22 & & & 68 & 64 & & & 41 & 44 & & & & & & \\
\hline Swaziland & 98 & 99 & 96 & 99 & 90 & 98 & 78 & 86 & 89 & 89 & 88 & 88 & 88 & 67 & 98 & 98 & & & 36 & & & \\
\hline Togo & 86 & 79 & 92 & 91 & 88 & 87 & 85 & 82 & & & 81 & 81 & 86 & 34 & 88 & 85 & & & 85 & 35 & 85 & 82 \\
\hline Uganda & 93 & 93 & 89 & 89 & 78 & 78 & 82 & 82 & & & 85 & 85 & 66 & 50 & 82 & 82 & & & & & & \\
\hline
\end{tabular}




\begin{tabular}{|c|c|c|c|c|c|c|c|c|c|c|c|c|c|c|c|c|c|c|c|c|c|c|}
\hline \multirow{2}{*}{ Country } & \multicolumn{2}{|c|}{ BCG } & \multicolumn{2}{|c|}{ Penta 1} & \multicolumn{2}{|c|}{ Penta 3} & \multicolumn{2}{|c|}{ MCV1 } & \multicolumn{2}{|c|}{ MCV2 } & \multicolumn{2}{|c|}{ PAB } & \multicolumn{2}{|c|}{ PCV3 } & \multicolumn{2}{|c|}{ OPV3 } & \multicolumn{2}{|c|}{ RCV1 } & \multicolumn{2}{|c|}{ Rota Last } & \multicolumn{2}{|c|}{ YFV } \\
\hline & 2015 & 2014 & 2015 & 2014 & 2015 & 2014 & 2015 & 2014 & 2015 & 2014 & 2015 & 2014 & 2015 & 2014 & 2015 & 2014 & 2015 & 2014 & 2015 & 2014 & 2015 & 2014 \\
\hline $\begin{array}{l}\text { United Republic of } \\
\text { Tanzania }\end{array}$ & 99 & 99 & 99 & 99 & 98 & 97 & 99 & 99 & 57 & 29 & 90 & 88 & 95 & 93 & 96 & 97 & 99 & 99 & 98 & 97 & & \\
\hline Zambia & 99 & 99 & 97 & 96 & 90 & 86 & 90 & 85 & 47 & 33 & 85 & 85 & 86 & 77 & 90 & 78 & & & 82 & 73 & & \\
\hline Zimbabwe & 90 & 99 & 94 & 98 & 87 & 91 & 86 & 92 & & & 75 & 75 & 87 & 91 & 88 & 92 & & & 87 & 48 & & \\
\hline
\end{tabular}

Analysis of 2015 JRF data indicates that 43 countries in the region confirmed having a budget line for vaccine procurement. Seven countries reported having funded $>90 \%$ of their vaccine costs. Thirty countries are funding $<50 \%$ of the vaccine cost by their national governments.

The situation is completely different for the total funding of routine immunization activities funded by national Governments. Only five countries have funded $100 \%$ of their routine immunization costs and 28 countries funded $<50 \%$. Eight countries did not provide this information in their 2015 JRF.

\section{Discussion}

The GVAP plans recommend countries to achieve a national coverage $\geq 90 \%$ for the third dose of DTP3. The analysis of the 2015 WUENIC data shows that only 13 out of 47 countries $(27.6 \%)$ have been able to meet the targeted DPT3 coverage for three consecutive years, while 16 out of 47 reached the target in 2015. The surviving infants living in those 13 countries represent only $12 \%$ of the total regional target population. The majority of surviving infants in the region still live in countries $(34 / 47)$ which did not reach the GVAP/RISP recommended target of $\geq 90 \%$ coverage at the national level, which indicates persistent inequities between countries. Five years of the implementation of GVAP 2011-2020 call on more efforts to meet and sustain the momentum towards meeting the set goals. Some countries have however met the GVAP objective on the relevant antigens or were on track for 2015 (see Table 1). Overall, the Region appears to be off track and needs remedial and urgent actions to reach all children with effective vaccine and ensure equity in the coverage.

\section{Challenges for reaching every child}

The challenges facing countries are better appreciated under the different immunization components. For instance, for programme management, monitoring, and accountability, it is observed that there is fragmentation in planning and lack of clear leadership. These factors create gaps in micro-planning and denominator figures (target population issues); gaps in health information and monitoring systems as well as constraints in data quality management, archiving and analysis. There are challenges of ineffective use and interpretation of data to redirect the programme.
Improvements in immunization spending in most African countries have predominantly been due to donor funds. However, of the countries that established line items in their national budgets for routine vaccines, over a third of them did not fund the programmes, and those that had drawn financial plans did not utilize them to the degree expected.

Another challenge is the quality of immunization data in many countries in the Region. Various external evaluations have identified many inconsistencies in reported data suggesting that immunization data monitoring remains weak in most African countries?.

Service delivery also suffers a range of shortcomings in the form of insufficient supply and access to quality services, limited service delivery points, and outreach sites. The shortcoming in service delivery is closely related to the inadequate use of the Gavi Alliance health system strengthening funds to strengthen routine immunization activities and is exacerbated with security constraints in some countries of the region ${ }^{6}$.

Another set of challenges has to do with logistic and cold chain issues. Insufficient storage capacity at central and intermediate levels (high proportion of equipment failure or inappropriate) constituted a challenge to the ready availability of vaccines. There also inadequate supply and logistics systems resulting in recurrent shortages or overstock of vaccines and essential commodities as well as lack of funding for vaccine distribution at the most peripheral levels. Cold chain management in resourcepoor settings, where electricity is non-existent or erratic, coupled with a lack of adequate trained staff to administer vaccines present major challenges in most African countries. Furthermore, of those children who do receive the vaccines, some receive them late or at inappropriate timing, and likely receive sub-optimal disease protection.

Furthermore, weak communication strategies at all levels; insufficient demand creation, weak links with communities and their leaders and low community awareness and participation have all been identified as impediments to attaining the GVAP objectives. The GVAP recognized these and highlighted the need to promote community participation to engender demand for immunization services. In one of its targets, it was stated that individuals and communities should understand the values of vaccines and demand same as right and recognize 
their responsibilities too. Unfortunately, this seems to be lacking. The principle of country ownership also needs to be promoted. The analysis here reveals that very few countries provide funding for immunization.

\section{Recommendations}

As we pursue the targets of the Sustainable Development Goals (SDGs), the Region is cognizant of the various constraints and challenges to reaching every child. Since assuming office as the Regional Director, Dr. Moeti, has taken steps to ensure that these challenges are addressed and that the goal of equity in access to health is achieved in the Region. The Ministerial Conference held in February 2016 in Addis Ababa provided an opportunity to assess immunization programmes in Africa and the role of the government critically, take ownership of the Regional and country-specific problems, and develop precise strategies to overcome the challenges identified. The underlying theme for this ministerial event was to foster country ownership for sustainable funding to achieve the GVAP goals and targets while advocating for greater engagement with communities, civil society organizations and other partners for sustainable demand for immunization.

One of the key gaps recognized at the conference is the need to commit government resources for sustainable high and equitable immunization coverage. Thus the conference culminated in the signing of the Addis Declaration on Immunization (ADI) by ministers across Africa, committing them to 10 specific goals to achieve universal access to immunization in Africa. There is a need for increased immunization systems strengthening, as many are plagued by weak infrastructure and shortage of skilled human resources. More affordable and adapted vaccines need to be made available. Other key actions include strengthening the integration with other child survival and high impact interventions and extend the benefits of immunization to adolescents and adults.

The new Regional Strategic Plan for Immunization 2014-2020 which is intended to provide policy and programmatic guidance to Member States to optimize immunization services, in line with the GVAP, will be used to strengthen their immunization programmes further. Key approaches in the Regional Strategic Plan include integrating immunization into national health policy and plan and during emergencies, strengthening immunization financing, enhancing partnerships, building national capacity, improving monitoring and data quality, improving vaccine management, safety and regulation and promoting implementation, research, and innovations.

In conclusion, while commending countries in the African Region for giant steps made in EPI performance over the past four decades, there exist wide inter- and intracountry differences, with a significant number of children remaining unreached, un/under-vaccinated, and still dying from VPDs. Immunization systems' strengthening is essential in the framework of the overall health system strengthening, as most health facilities are under-staffed with inadequate resources to function efficiently. Issues of vaccine supply, financing, and sustainability require urgent attention. Increased political and financial commitment from governments as well as coordinated national evidence-informed efforts by all immunization stakeholders are needed to both maintain current achievements and make additional progress for EPI in the African Region. African leaders must be held accountable for meeting agreed country targets and honouring international commitments they have signed on to.

\section{References}

1. Larson HJ, Cooper LZ, Eskola J, et al. Addressing the vaccine confidence gap. Lancet. 2011; 378: 526-35.

2. Healy CM, Pickering LK. How to communicate with vaccine-hesitant parents. Pediatrics. 2011; 127: S127-S133.

3. Centers for Disease Control and Prevention Vaccine preventable deaths and the Global Immunization Strategy, 2006-2015. MMWR Morb Mortal Wkly Rep. 2006; 55: 511-515.

4. Bloom D. The value of vaccination. Adv Exp Med Biol. 2011; 697: 1-8.

5. Mavimbe JCT, Muquingue HN, Braa J, et al. Immunization coverage in Mozambique: From concepts to decision-making. Health Policy 2006; 79: 92-100.

6. Batt K, Fox-Rushby JA, Castillo-Riquelme M. The costs, effects and cost-effectiveness of strategies to increase coverage of routine immunizations in low- and middle-income countries: systematic review of the grey literature. Bulletin of the World Health Organization. 2004; 82(9): 689-96.

7. Moeti M. Immunization in the African Region. African Health Monitor. 2015; (19): 1 .

8. MachingaidzeS, Wiysonge CS, Hussey GD. Strengthening the Expanded Programme on Immunization in Africa: Looking beyond 2015. PLoS Med. 2013; 10(3): e1001405. doi:10.1371/journal.pmed.1001405

9. World Health Organization. WHO Vaccine Preventable Diseases Monitoring System http://apps.who.int/immunization_monitoring/ en/globalsummary/countryprofileselect.cfm. Accessed 31 March 2013. (Data as of July 2011.)

10. Wiysonge CS, Waggie Z, Rhoda L, et al. Vaccines for Africa (VACFA) website - an innovative immunisation advocacy tool. SAMJ. 2009; 99(5): 275.

11. Okeibunor JC, Ota MC, Akanmori BD, et al. Polio eradication in the African Region on course despite public health emergencies. Vaccine. 2015. http://dx.doi.org/10.1016/j.vaccine.2015.08.024

12. Arevshatian L, Clements CJ, Lwanga SK, et al. An evaluation of infant immunization in Africa: Is a transformation in progress. Bull World Health Organ. 2007; 85: 449-457. doi: 10.2471/BLT.06.031526.

13. Global Polio Eradication Initiative (GPEI). Infected countries. Available: http://www.polioeradication.org/Infectedcountries.aspx. Accessed 27 August 2012. 